\title{
I 8 months follow up of diastemas closure using ceramic veneers reinforced by lithium disilicate: a
} case report

\begin{abstract}
The use of ceramic veneers for the esthetic treatment of anterior teeth is favorable nowadays. They are considered as one of the most conservative treatment option for diastemas closure. The preservation of the tooth structure, color and contour stability, maintaining teeth vitality as well as predictable end results are among the advantages of ceramic veneers. The aim of this case report is to describe the treatment of diastemas using ceramic veneers reinforced by lithium disilicate.
\end{abstract}

Keywords: conservative preparation, diastema closure, esthetic dentistry, laminated ceramic veneers, lithium disilicate.
Volume 8 Issue 3 - 2017

\section{Mashael Bin Hasan}

Department of Restorative Dental Sciences, King Saud University, Saudi Arabia

\section{Correspondence: Mashael Bin Hasan, Department of Restorative Dental Sciences, Collage of Dentistry, King Saud University, P.o.Box 89606, Riyadh, I I692, Saudi Arabia, Emaildr_binhassan@yahoo.com}

Received: September 21, 2017 | Published: September 27 2017

\section{Introduction}

The presence of diastemas is one of the factors that affect the smile esthetics as it is occasioned by differences in tooth size. ${ }^{1}$ Composite resin and indirect ceramic restorations are two excellent treatment options for diastema closure depending on the size of the diastema. ${ }^{2,3}$ Although direct composite restorations have the advantages of conservation of the tooth structure, low cost, reversibility, and a relatively simple technique, ${ }^{4}$ it was reported that they have a high failure rate. ${ }^{5}$ for extensive tooth reconstruction due to secondary caries, loss of restoration, pigment impregnation, fracture, marginal defects, ${ }^{6}$ or a high degree of color instability. ${ }^{7}$ With the increased esthetic demands and the improvement in dental materials, the use of all-ceramics for indirect esthetic restorative procedures in recent years has increased. ${ }^{8,9}$ The introduction of ceramic veneers was in $1983 .{ }^{10}$ Since then, veneers have been one of the most viable treatment modalities as it is a conservative operative treatment procedure. ${ }^{11}$ Tooth preparation involves the removal of less than half the thickness of the enamel, preserving the tooth structure and leaving the rest enamel intact before veneer placement. ${ }^{10}$ which also gives the advantage of a reliable bonding to enamel surface. Moreover, Ceramic veneers have the advantages of color and contour stability, superior esthetics, ${ }^{12}$ maintaining tooth vitality, and producing predictable results. ${ }^{13-15}$, having failure rates of only $0 \%$ to $5 \%$ over 1 to 5 years. ${ }^{16}$ Furthermore, they have compressive strength, surface smoothness, abrasion resistance, gloss, and low plaque accumulation. ${ }^{17,18}$ Many ceramic types are available such as feldspathic ceramic, leucitereinforced glass ceramics and lithium-disilicate restorative material. Lithium disilicates are glass ceramics that are currently recommended for porcelain laminate veneers (PLVs) due to the ability to produce thin layers, thereby making them more translucent and esthetic. ${ }^{19}$ this manuscript reports a case describing the treatment of diastema and spaces closure of maxillary incisors using lithium disilicatereinforced ceramic veneers.

\section{Case description}

\section{Diagnosis and treatment planning}

A-33-year old female patient presented to the restorative clinics at the College of Dentistry, King Saud University, Saudi Arabia. She expressed her desire to find a long-term treatment for the gaps between her front teeth. The patient reported an orthodontic treatment that failed to close the spaces and that direct composite restorations were used to close them 10years ago. During this period of time, the patient reported that the restorations got stained frequently and needed to be polished regularly, some of them got chipped, and others got broken and needed to be repaired or re-done. Oral examination revealed the presence of diastemas between the maxillary anterior teeth, multiple old chipped, broken and stained composite restorations (Figures 1-3). After examination, radiographic and photographic data was collected from the patient, alginate impressions (United Alginate, Major Prodotti Dentari SpA, Moncalieri, Italy) were taken for diagnostic models. The models were then mounted on a semi-adjustable articulator. They were studied to decide the shape and size of the restorations with the help of a diagnostic wax-up which was presented to the patient the following appointment, and any desired modification by the patient was adjusted. The patient was provided with the option of ceramic veneers for the maxillary arch, while bleaching was recommended for the mandibular arch because they are less visible. The final treatment plan was approved. A transparent template was done on the wax-up on the study models to be used in the fabrication of the provisional veneers later on.

\section{Dental preparation and impression}

Shade selection was done prior to the bleaching procedure. Teeth prophylaxis was done using pumice. The teeth were then bleached (inoffice bleaching) using 35\% hydrogen peroxide (Zoom! whitespeed, Philips-USA). After 2 weeks, the shade was evaluated and the patient 
was satisfied with the results as the shade changed from (A2) to (BL4). A conservative preparation of the enamel was performed from the right first premolar (\#14) until the left one (\#24). A $0.3 \mathrm{~mm}$ shallow chamfer finish line was prepared for the ceramic veneers using the depth cutting diamond bur (DIATECH, Dübendorf, Switzerland). The surface was then smoothened using the long tapered coarse diamond bur followed by the fine one (DIATECH, Dübendorf, Switzerland) (Figure 4). At the same appointment, gingival displacement was obtained using retraction cords ( \#00, Ultradent, South Jordan UT, USA) and the final impression was done, using silicone impression material (Aquasil impression material, Dentsply, International Inc. Canada). The ceramic shade was designated as BL4. The incisal characterization was made with blue phase and opaque line. The glass-ceramic lithium disilicate was used (IPS e.max Press, Ivoclar Vivadent Schaan, Liechtenstein, Zurich). Temporary veneers were then fabricated using the transparent template and Protemp 4 provisional material (3M ESPE St. Paul, MN, and USA). The provisional veneers were cemented with Temp-Bond NE (Kerr, Orange, CA) temporary cement (Figure 5).

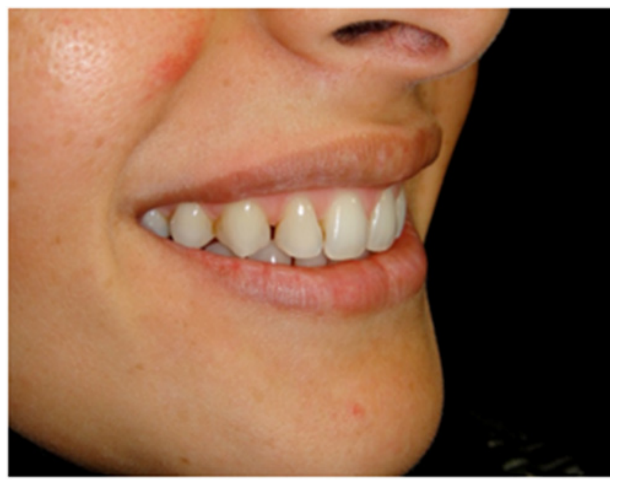

Figure I Preoperative patient's smile.

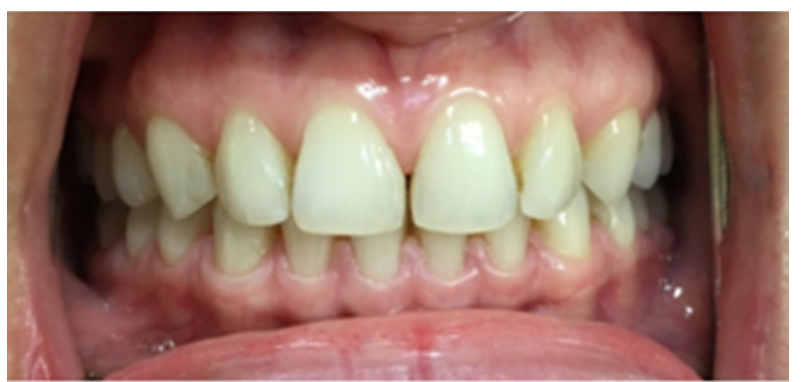

Figure 2 Preoperative intra-oral front view.

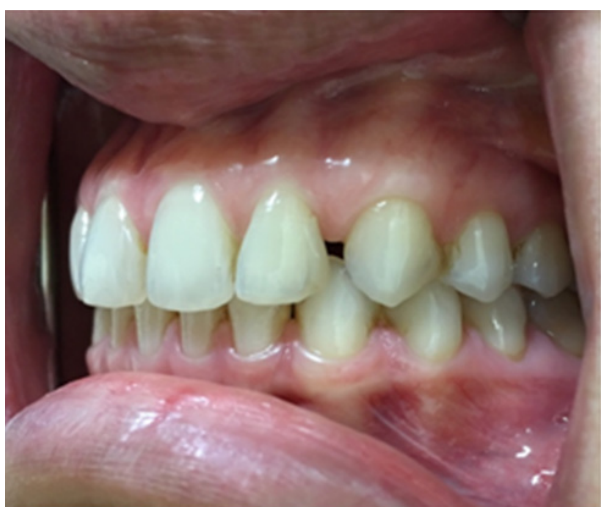

Figure 3 Preoperative left side view.

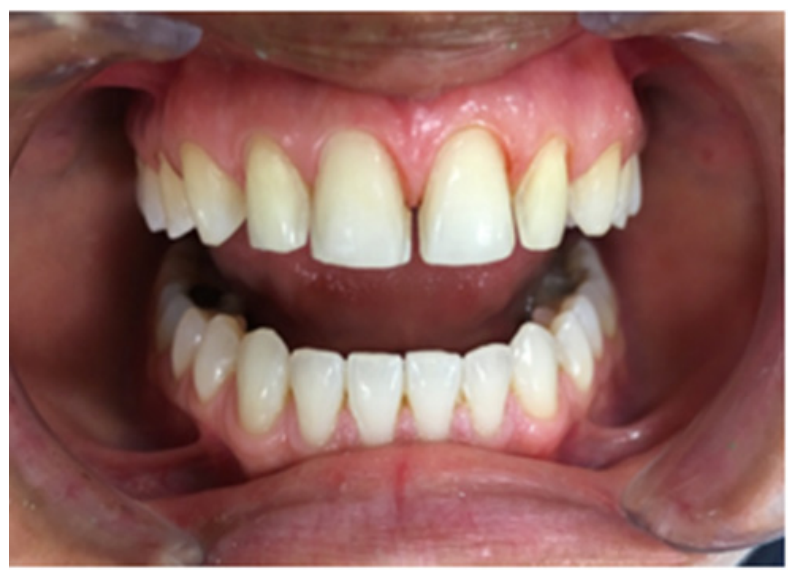

Figure 4 Teeth preparation.

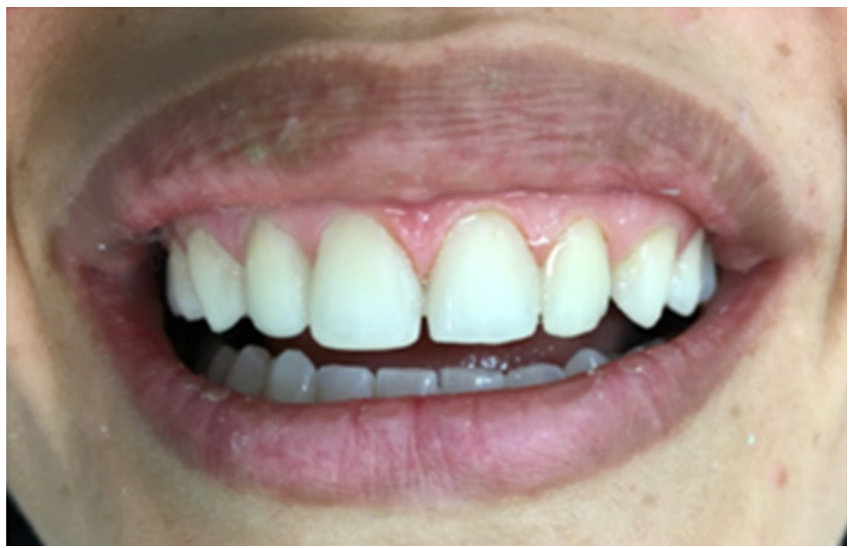

Figure 5 Provisional restorations.

\section{Veneers try in and cementation}

The prepared teeth were cleaned and the veneers were tried-in. The marginal adaptation, proximal contacts, shade and alignment of the veneers as well as the periodontal relation were checked. The cementation was done using dual cure resin cement Veriolink N (Ivoclar Vivadent, Schaan, Lichtenstein) the internal surfaces of the veneers were etched with $9.5 \%$ hydrofluoric acid for 30 seconds (Condicionador de Porcelanas, Dentsply Brasil). The surfaces were washed with water, and dried with an air syringe. The veneers were silanized with a silane coupling agent (Monobond Plus, Ivoclar Vivadent) that was applied and left to react for $60 \mathrm{~s}$ before air drying. The enamel was conditioned with $37 \%$ phosphoric acid for 30 seconds (Total Etch, Ivoclar Vivadent, Schaan, Lichtenstein) then washed for another 30s and gently dried. The adhesive agent (ExciTE F DSC, Ivoclar Vivadent, Schaan, and Lichtenstein)) was applied on both the enamel (for 10s using a micro-brush) and the inner surface of the veneers. Excess bonding was blown to a thin film using air spray. Then the catalyst and base of the dual resin cement were mixed and applied on the inner surface of the veneers that were then seated on the teeth. The cementation procedure was performed one-by-one starting with the central incisors, laterals, canines and finally the premolars. Excess cement was removed with a brush, and each surface was photo activated for 60seconds using LED-curing unit (Blue phase LED-curing light, Ivoclar Vivadent, Schaan, Lichtenstein).It was then removed after curing using a \#12 surgical blade. Flossing was performed at the proximal areas to ensure interproximal contact patency. Occlusal contacts were marked, and protrusive and lateral 
movements were checked. The final appearance is shown in Figures 6-8. Follow up was done after 18 months (Figure 9).

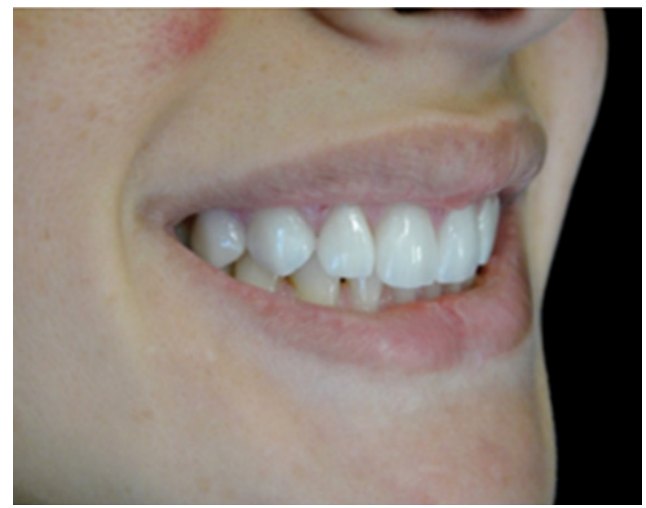

Figure 6 Post-operative patient's smile.

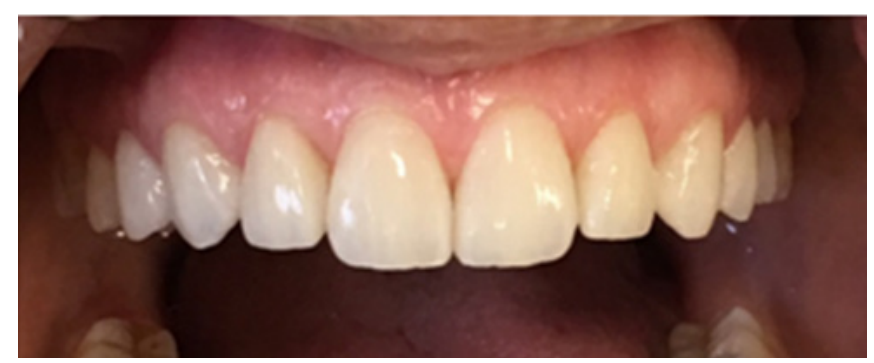

Figure 7 Post-operative intraoral front view.

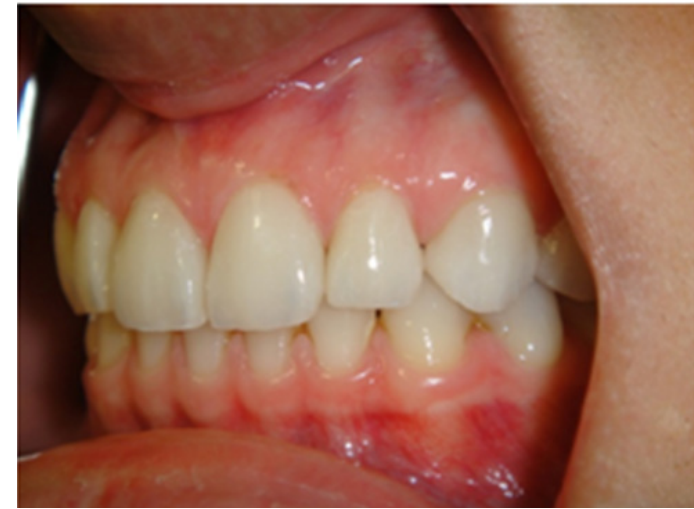

Figure 8 post-operative intraoral left side view.

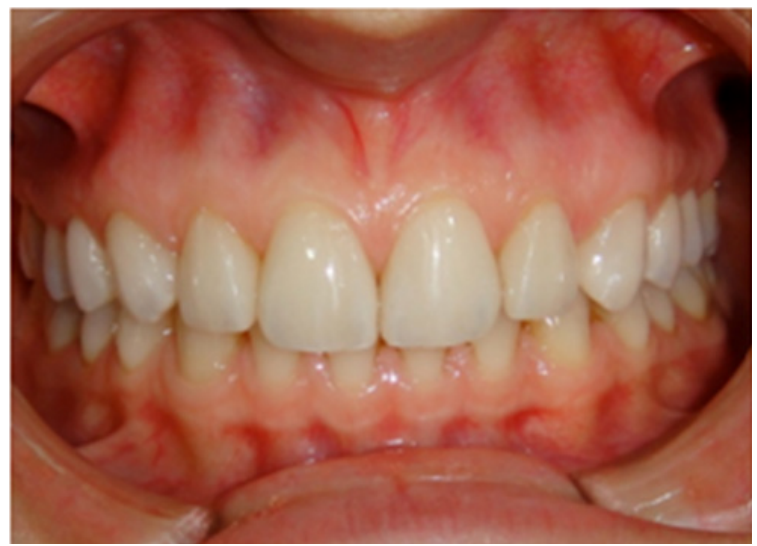

Figure 9 I8months follow up visit.

\section{Discussion}

Microdontia is one of the causes of diastimas and spaces between the teeth, which adversely affect the esthetics. ${ }^{20}$ The present case report justifies the choice of diastema closure with ceramic veneers as the patient was not happy with the esthetics and the continuous repairing of the previous composite restorations used to close the diastima. Proper examination, diagnosis and treatment plan as well as good oral hygiene are mandatory for a successful treatment of anterior diastemas. ${ }^{21,22}$ Study models and wax-ups are important to evaluate clinical conditions, restoration form, occlusal factors, and esthetic design..$^{23}$ Giving the fact that lithium disilicate ceramic veneers have relatively high wear resistance without jeopardizing optical properties, they were fabricated with thicknesses of $0.3 \mathrm{~mm}$ as the procedure was intended to be conservative and minimally invasive. ${ }^{24}$

\section{Conclusion}

Restoring of smile esthetics of a patient with diastemas with ceramic veneers proved to be effective with conservative preparations. Detailed planning and proper selection of dental materials contribute to the successful treatment.

\section{Acknowledgments}

None.

\section{Conflicts of interest}

I wish to confirm that there are no known conflicts of interest associated with this publication and there has been no significant financial support for this work that could have influenced its outcome.

\section{Funding}

None.

\section{References}

1. Wolff D, Kraus T, Schach C, et al. Recontouring teeth and closing diastemas with direct composite buildups: a clinical evaluation of survival and quality parameters. $J$ Dent. 2010;38(12):1001-1009.

2. De Araujo Júnior EM, Baratieri LN, Monteiro Júnior S, et al. Direct adhesive restoration of anterior teeth: Part 3. Procedural considerations. Pract Proced Aesthet Dent. 2003;15(6):433-437.

3. Baratieri LN, Araujo EM, Monteiro S. Composite restorations in anterior teeth: fundamentals and possibilities. Quintessence, USA. 2005;3-82.

4. Heymann HO, Hershey HG. Use of composite resin for restorative and orthodontic correction of anterior interdental spacing. J Prosthet Dent. 1985;53(6):766-771.

5. Tuncer D, Yazici A, Ozgünaltay G, et al. Clinical evaluation of different adhesives used in the restoration of non-carious cervical lesions: 24 month results. Aust Dent J. 2013;58(1):94-100.

6. Kopperud SE, Tveit AB, Gaarden T, et al. Longevity of posterior dental restorations and reasons for failure. Eur J Oral Sci. 2012;120(6):539-548.

7. Garoushi S, Lassila L, Hatem M, et al. Influence of staining solutions and whitening procedures on discoloration of hybrid composite resins. Acto Odontol Scand. 2013;71(1):144-150.

8. Walter RD, Raigrodski AJ. Critical appraisal: clinical considerations for restoring mandibular incisors with porcelain laminate veneers. $J$ Esthet Restor Dent. 2008;20(4):276-281. 
9. Guess PC, Stappert CF, Strub JR. Preliminary clinical results of a prospective study of IPS e.max Press-and Cerec ProCAD- partial coverage crowns. Schweiz Monatsschr Zahnmedl. 2006;116(5):493-500.

10. Pini NP, Aguiar FH, Lima DA, et al. Advances in dental veneers: materials, applications, and techniques. Clin Cosmet Investig Dent. 2012;4:9-16.

11. Heffernan MJ, Aquilino SA, Diaz-Arnold AM, et al. Relative translucency of six all-ceramic systems. Part II: core and veneer materials. J Prosthet Dent. 2002;88(1):10-15.

12. Rotoli BT, Lima DA, Pini NP, et al. Porcelain veneers as an alternative for esthetic treatment: clinical report. Oper Dent. 2013;38(5):459-466.

13. Edelhoff D, Sorensen JA. Tooth structure removal associated with various preparation designs for anterior teeth. $J$ Prosthet Dent. 2002;87(5):503-509.

14. Zhang F, Heydecke G, Razzoog ME. Double-layer porcelain veneers: effect of layering on resulting veneer color. J Prosthet Dent. 2000;84(4):425-431.

15. Jordan A. Clinical aspects of porcelain laminate veneers: considerations in treatment planning and preparation design. $J$ Calif Dent Assoc. 2015;43(4):199-202.

16. Peumans M, Van Meerbeek B, Lambrechts P, et al. Porcelain veneers: a review of the literature. J Dent. 2000;28(3):163-177.
17. Seghi RR, Sorensen JA. Relative flexural strength of six new ceramic materials. Int J Prosthodont. 1995;8(3):239-246.

18. McLaren EA. All-ceramic alternatives to conventional metal-ceramic restorations. Compend Contin Educ Dent. 1998;19(3):307-326.

19. Shenoy A, Shenoy N. Dental ceramics: An update. J Conserv Dent. 2013;13(4):195-203.

20. Namdar F, Atasu M. Macrodontia in association with a contrasting character microdontia. J Clin Pediatr Dent. 1999;23(3):271-274.

21. Huang WJ, Creath CJ. The midline diastema: a review of its etiology and treatment. Pediatr Dent. 1995;17(3):171-179.

22. Chu $\mathrm{CH}$, Zhang $\mathrm{CF}$, Jin LJ. Treating a maxillary midline diastema in adult patients: a general dentist's perspective. $J$ Am Dent Assoc. 2011;142(11):1258-1264.

23. Rudd KD. Making diagnostic casts is not a waste of time. J Prosthet Dent. 1968;20(2):98-100.

24. Conrad HJ, Seong WJ, Pesun IJ. Current ceramic materials and systems with clinical recommendations: a systematic review. J Prosthet Dent. 2007;98(5):389-404. 